\title{
Traumatología infantil y COVID-19. Experiencias en pandemia desde Córdoba, Argentina
}

\author{
Antonio E. Pedro Maraude, Melina Luna, Lorena Zecchini \\ Hospital de Niños de la Santísima Trinidad, Córdoba, Argentina
}

\begin{abstract}
RESUMEN
Introducción: La enfermedad por coronavirus se expandió rápidamente, esto llevó a un aumento de la demanda de los servicios sanitarios, por lo cual fue necesario adaptarse de manera acorde. Nuestro objetivo es proporcionar una visión general del impacto en la atención y de nuestra experiencia, identificar aspectos positivos y aquellos por mejorar para futuras planificaciones. Materiales y Métodos: Se registraron el número de consultas diarias, la edad, el sexo, la presencia de traumatismo, el lugar de la lesión, el motivo de consulta, el diagnóstico y el tratamiento. Se comparó la cantidad de atenciones con las flexibilizaciones y con igual período de 2019. Resultados: Se realizaron 120 consultas, 33 procedimientos quirúrgicos, 185 atenciones por guardia, 160 con traumatismo. El lugar más frecuente de traumatismo fue el domicilio (56,25\%). El 30\% eran fracturas de muñeca y el $28,8 \%$, supracondíleas. Las consultas aumentaron con las flexibilizaciones del confinamiento. Conclusiones: Es fundamental adaptarse rápido teniendo en cuenta que las fracturas comunes seguirán ocurriendo, pese al confinamiento al igual que las infecciones osteoarticulares. No descuidar tampoco la enfermedad crónica impostergable.
\end{abstract}

Palabras clave: Traumatología infantil; COVID-19; pandemia; ortopedia infantil; cuarentena; fracturas.

Nivel de Evidencia: III

Pediatric Traumatology and COVID-19. Pandemic Experiences from Córdoba, Argentina

\section{ABSTRACT}

Introduction: Coronavirus disease spreads rapidly, which leads to an increased demand for health services. Such services must be adapted accordingly. Our aim is to provide a general vision on the way these circumstances affect our practice, based on our experience and remarking the positive aspects as well as those which need to change in the future. Materials and Methods: $\mathrm{A}$ record was kept of the daily number of consultations, age of the patient, gender, trauma history, place of injury, reason for consultation, diagnosis and treatment. These data were contrasted with the records for the different quarantine phases and with the records for the same period of 2019. Results: The number of consultations amounts to 120, the number of surgeries amounts to 33 , and 185 emergency room consultations were carried out, 160 of them with trauma patients. The most frequent place of injury was the home (56.25\%). $30 \%$ of the injuries were wrist fractures and $28.8 \%$ were supracondylar fractures. The number of patients increased over quarantine flexibilization. Conclusion: Fast adaptation is fundamental. Common fractures occur even during quarantine, as well as infections. Professionals should avoid neglecting unpostponable attention to patients with a chronic condition.

Key words: Pediatric orthopedic; COVID-19; pediatric trauma; quarantine; fractures.

Level of Evidence: III

\section{INTRODUCCIÓN}

La enfermedad por el nuevo coronavirus (COVID-19) se expandió rápidamente desde los primeros contagios en Wuhan, China, hasta ser declarada pandemia por la Organización Mundial de la Salud, el 11 de marzo de 2020.

El contagio de persona a persona en grandes centros urbanos favoreció una rápida propagación del virus, ${ }^{1}$ lo que llevó a un aumento de la demanda de los servicios sanitarios y a su colapso en algunos países. ${ }^{2}$

Por supuesto, cada país e incluso cada ciudad experimenta, de manera diferente, los brotes en función de su preparación, capacidades, recursos e intervenciones implementadas.

Recibido el 30-6-2020. Aceptado luego de la evaluación el 25-9-2020 • Dr. ANTONIO E. PEDRO MARAUDE • apedromaraude@ gmail.com

Cómo citar este artículo: Pedro Maraude AE, Luna M, Zecchini L Traumatología infantil y COVID-19. Experiencias en pandemia desde Córdoba, Argentina. Rev Asoc Argent Ortop Traumatol 2021:86(1):122-129. https://doi.org/10.15417/issn.1852-7434.2021.86.1.1147 
Se estima que las comunidades y los entornos de atención médica deberán adaptarse durante algunos años. ${ }^{3}$

Una de las medidas adoptadas y que demostró mayor efectividad a la hora de disminuir la propagación viral fue el aislamiento social con la consiguiente interrupción de las actividades no esenciales.

Es probable que, en países en cuarentena, haya menos trauma, pero los accidentes y las lesiones osteoarticulares siguen ocurriendo. ${ }^{2,3}$

Junto con esto, es necesario adaptar el sistema de salud, modificando la atención habitual con el fin de reducir el riesgo de los pacientes, los familiares y los trabajadores de la salud, siempre teniendo en cuenta no descuidar la atención de otros cuadros y haciendo hincapié en el balance riesgo/beneficio de retrasar la atención de enfermedades habituales. ${ }^{2}$

En la Argentina, el 19 de marzo, se decretó el "Aislamiento Social, Preventivo y Obligatorio" que fue sumamente estricto las primeras dos semanas y ha ido sufriendo extensiones con modificaciones y flexibilizaciones que aumentaron progresivamente la circulación de personas.

Según informa el Ministerio de Salud, hasta el 14 de junio, se han diagnosticado 31.564 casos de COVID-19, la mayoría en la Ciudad Autónoma de Buenos Aires y el Área Metropolitana de Buenos Aires. ${ }^{4}$

La provincia de Córdoba, segunda provincia con mayor población del país (INDEC, censo 2010) había confirmado 495 casos en el mismo período, en su gran mayoría, en la ciudad capital.

Desde el inicio de la pandemia y su curso por diferentes países, se han recomendado y se han reportado experiencias en cuanto a la adaptación de los Servicios de Ortopedia y Traumatología para colaborar con el sistema sanitario y brindar atención a la demanda que se genere.

Como especialistas en Ortopedia y Traumatología infantil, que se desempeñan en una institución de tercer nivel de atención, elegida como centro de referencia para pacientes con COVID-19, tuvimos que diagramar protocolos de atención en emergencias, consulta externa y cirugía, con el objetivo de dar respuesta a la demanda que surgiera protegiendo siempre al paciente, a su familia y a nuestro propio equipo de salud.

El objetivo de este artículo es proporcionar una visión general del impacto en la atención y nuestra experiencia en este particular contexto, así como de la evaluación de los motivos de consulta, los mecanismos de trauma, las lesiones habituales y el tratamiento en épocas de Aislamiento Social, Preventivo y Obligatorio. El fin último es identificar aspectos positivos y aquellos por mejorar para futuras planificaciones en contextos de este tipo.

\section{MATERIALES Y MÉTODOS}

A partir del decreto de aislamiento y el inicio de la cuarentena, en el Servicio de Ortopedia y Traumatología, se tomaron diferentes decisiones con el propósito de mantener la atención de pacientes, por su urgencia o porque el retraso del tratamiento implicaría un deterioro importante del cuadro, y al mismo tiempo, proteger a todos los implicados en el proceso de una exposición innecesaria y de un posible contagio. Se registraron todas estas decisiones en un protocolo detallado y se generaron equipos de trabajo por guardia de 24 horas.

Se realizó el registro de consultas diarias (consultorio, cirugía y Guardia). En cuanto al consultorio, se tuvieron en cuenta la cantidad de consultas diarias y los motivos más frecuentes de consulta.

Con respecto a las atenciones por Guardia, se registró diariamente a los pacientes teniendo en cuenta la edad, el sexo, la presencia o no de traumatismo, el lugar del trauma, el mecanismo de trauma o el motivo de consulta, el diagnóstico y el tratamiento efectuado.

Toda esta información se recabó entre el 15 de abril y el 31 de mayo inclusive.

Se comparó la cantidad de pacientes atendidos con las flexibilizaciones y el aumento de la circulación. Asimismo, se comparó la cantidad de pacientes atendidos en igual período de 2019.

\section{RESULTADOS}

Se armaron cinco equipos de dos traumatólogos por día, cada equipo realizaba guardias de 24 horas, inclusive los fines de semana. A su vez, se generó un cronograma de refuerzos con el resto del personal para cubrir semanalmente de lunes a viernes (solo debían asistir en caso de cirugía pendiente del día anterior para cubrir la atención externa durante el procedimiento quirúrgico).

Se suspendieron los turnos programados por consultorios externos, pero estos permanecieron abiertos para atender los controles de fracturas, a pacientes posquirúrgicos y oncológicos.

En el período registrado, se atendió a 120 pacientes por consultorios externos. El $80 \%$ de esas atenciones fueron por control de fracturas y pacientes posquirúrgicos (algunos atendidos antes de la cuarentena). El porcentaje restante incluyó controles oncológicos (5\%), tratamientos previos (10\%) y consultas ortopédicas (5\%). 
En igual período de 2019, se realizaron 550 consultas por consultorios externos: el 60\% por control de fracturas y pacientes en el posoperatorio, y el $40 \%$ por problemas ortopédicos (columna 10\%, tumores $5 \%$, Neuroortopedia $5 \%$, cadera $10 \%$, otros $10 \%$ ).

De igual manera, se suspendieron las cirugías programadas. En el período estudiado, se realizaron 33 procedimientos quirúrgicos: $14(42,4 \%)$ por fracturas supracondíleas del codo; tres (9\%), por fracturas del cóndilo lateral; siete $(21,2 \%)$, por otras fracturas (una femoral). El resto fueron infecciones osteoarticulares y un recambio protésico tumoral por rotura del implante.

En 2019, siempre en el mismo período, se realizaron 118 procedimientos quirúrgicos entre cirugías programadas y urgencias.

Con respecto a la atención por Guardia, se asistió a 185 pacientes; el $62,2 \%$ eran varones $(\mathrm{n}=115)$ y el 37,8\%, niñas $(\mathrm{n}=70)$. La distribución por edad fue 89 pacientes $(48,1 \%)$ de 0 a 5 años, $68(36,7 \%)$ de 6 a 10 años y 28 $(15,1 \%)>10$ años. En el mismo período de 2019, se asistió a 935 pacientes por Guardia. No contamos con la discriminación de este período.

La cantidad y la progresión de pacientes por día se registran en la Figura 1. De ellos, 160 (86,5\%) tenían un traumatismo previo y $25(13,5 \%)$ no habían sufrido un traumatismo.

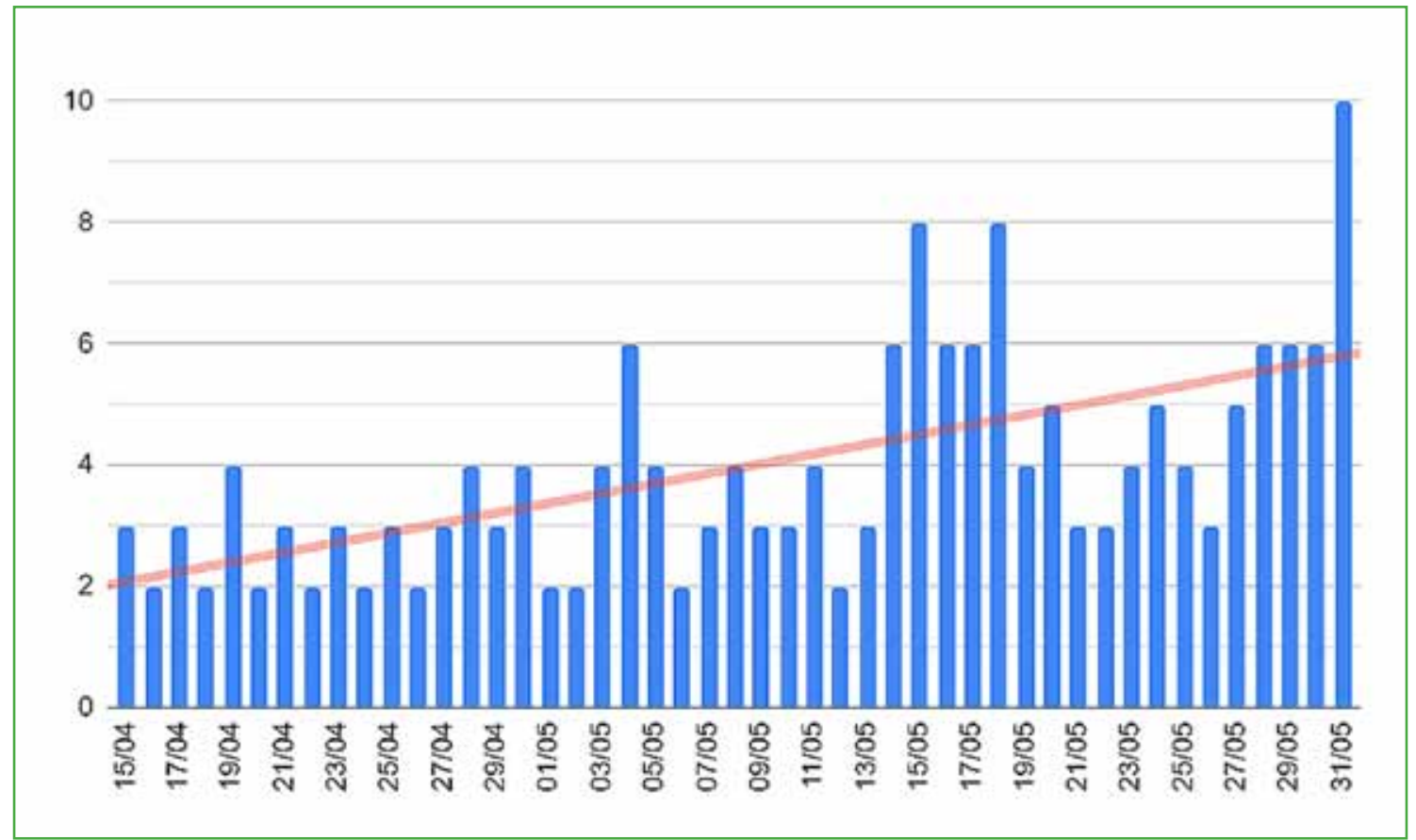

Figura 1. Progresión diaria de pacientes atendidos por Guardia en el Hospital de Niños de Córdoba, desde el 15/04/2020 hasta el 31/05/2020.

Los motivos de consulta se enumeran en la Tabla, el más frecuente fue caída de propia altura $(n=44)$. En la Figura 2, se detallan el lugar del traumatismo. 
Tabla. Motivos de consulta y mecanismo del trauma

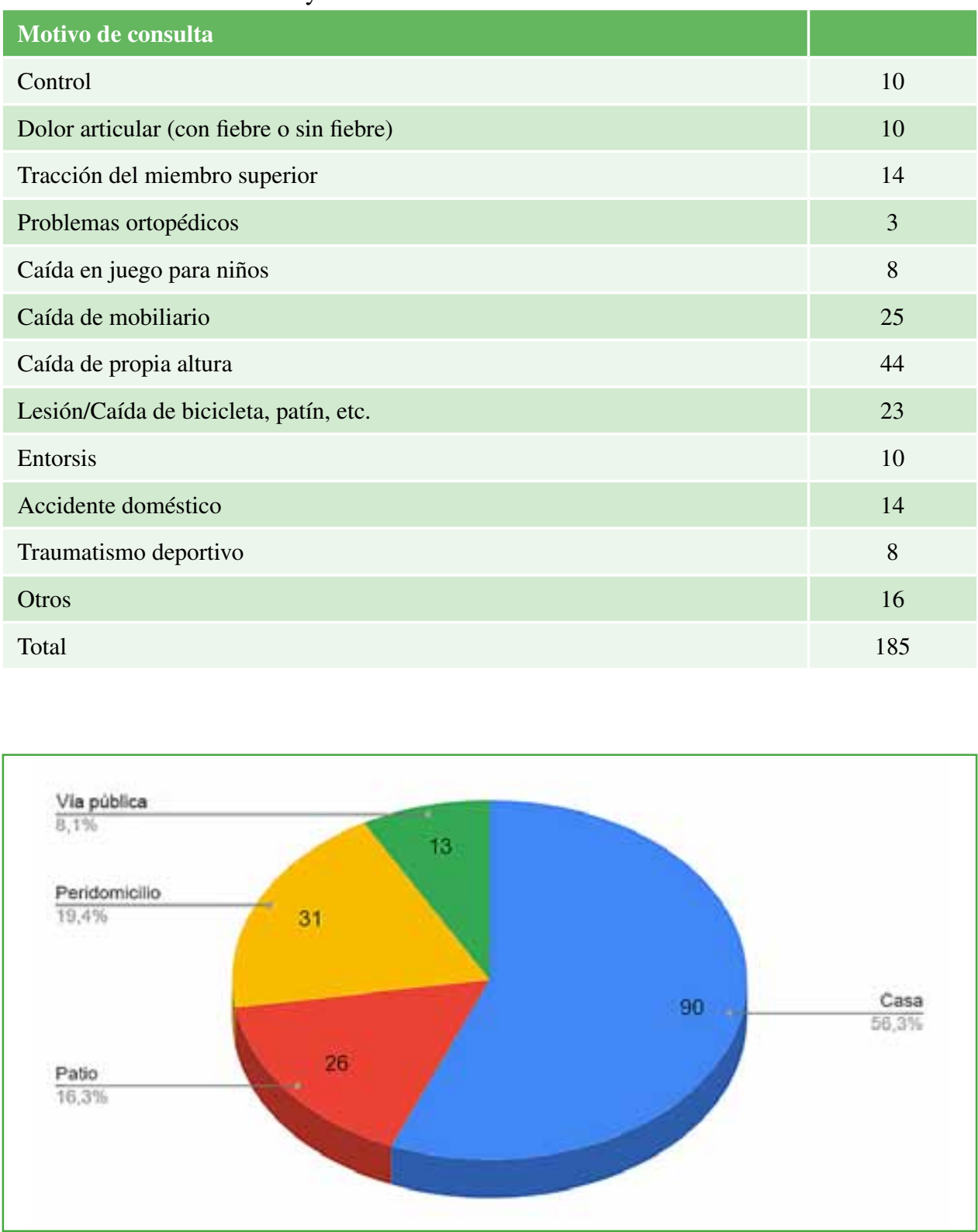

Figura 2. Lugar del traumatismo. Se entiende por peridomicilio a las inmediaciones, incluye vereda, vecino y plaza cercana.

El diagnóstico más frecuente fue, con gran diferencia, fractura de miembro superior $(43,25 \%, \mathrm{n}=80)$, seguido de traumatismos sin fractura tanto del miembro inferior $(n=25)$ como del miembro superior $(n=22)$. El resto de los diagnósticos se muestra en la Figura 3.

En la Figura 4, se detallan las fracturas de miembros superiores. En 2019, hubo 201 fracturas supracondíleas, lo que arroja un promedio de 16,75 por mes.

Con respecto a las fracturas de miembros inferiores, en el período estudiado, se trataron cinco fracturas de fémur, una de ellas quirúrgica y el resto tratadas con yeso. Además, hubo dos fracturas de tibia, una de maléolo medial, una de peroné y una fractura expuesta de calcáneo por accidente en motocicleta.

No hubo contagios entre el personal del Servicio ni fue necesario aislar a ninguno de los miembros del equipo. 


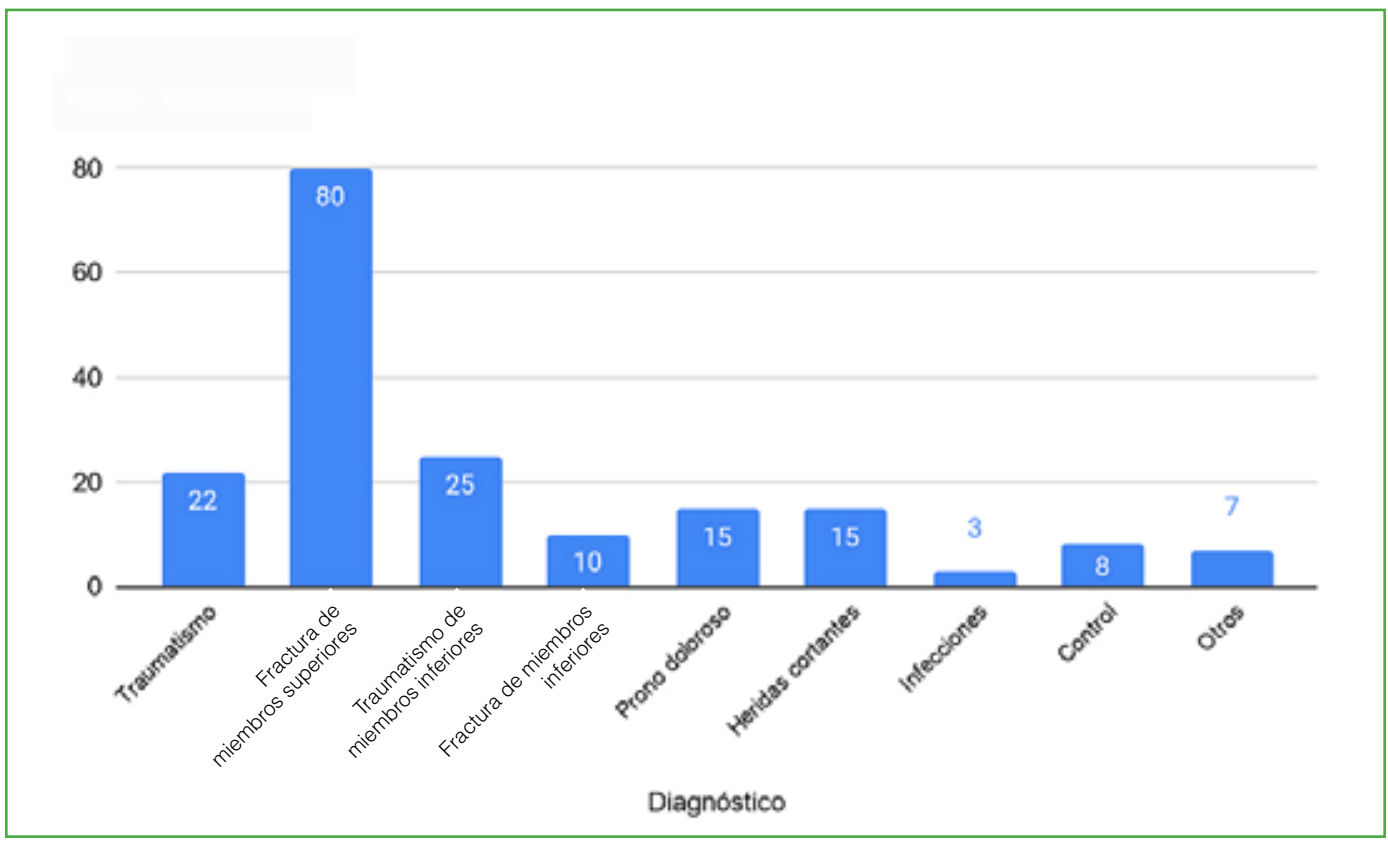

Figura 3. Cantidad por tipo de diagnósticos. "Otros" incluye refuerzo o retiro de yeso.

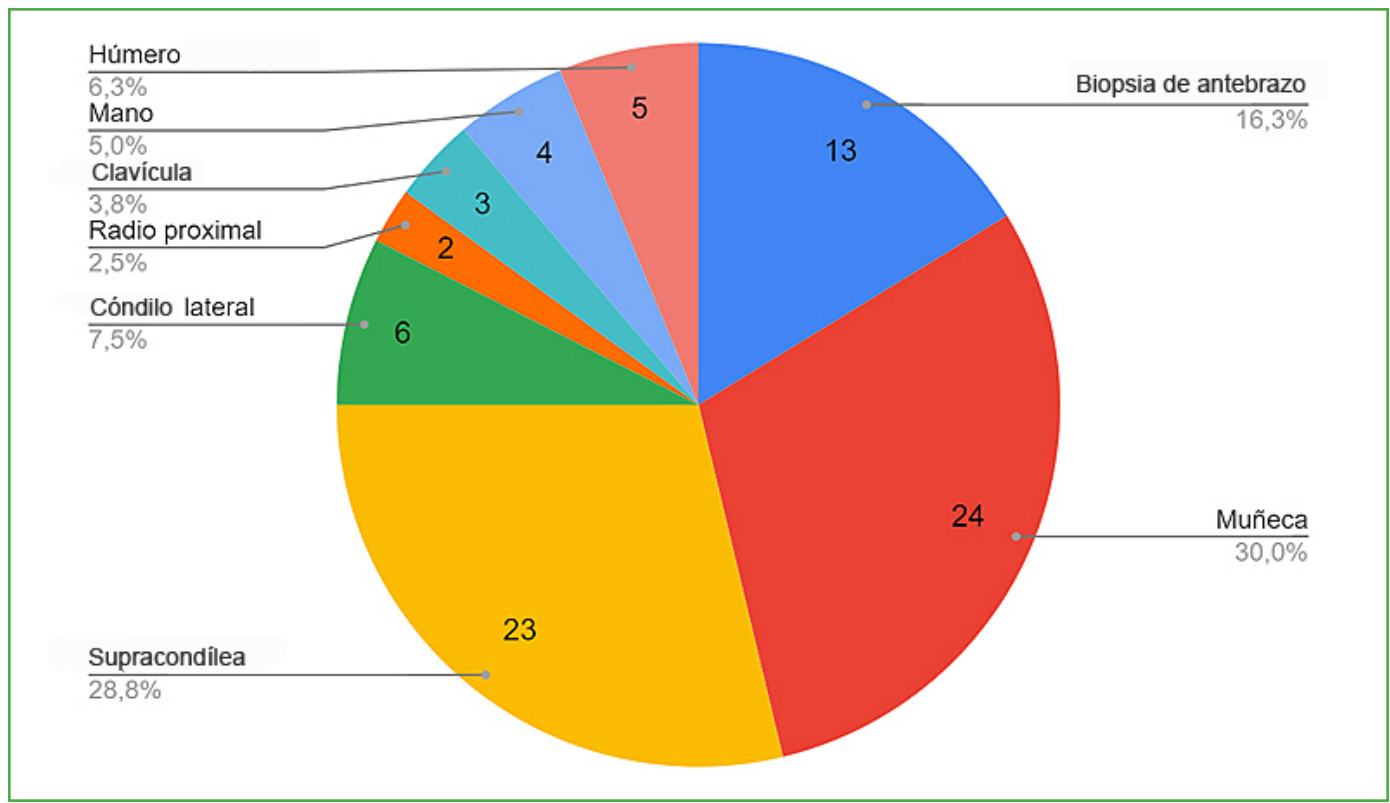

Figura 4. Fracturas de miembros superiores. Húmero incluye diafisarias y proximales.

\section{DISCUSIÓN}

Un contexto cambiante requiere rápida adaptación. Muchos Servicios debieron modificar sus esquemas de atención para dar respuesta a la demanda en tiempos de pandemia. Ortopedia y Traumatología no está exento y tiene la particularidad de que, pese a no atender directamente a pacientes comprometidos por COVID-19, debe estar preparado para recibir y tratar las urgencias. Se estima que, al disminuir la circulación, disminuye el trauma, pero aun así, sigue siendo un motivo de consultas en los servicios de emergencia. . $3,3-14^{-10}$ 
Adaptar el sistema implica proteger al personal, al paciente y a sus familiares en el proceso de atención de los enfermos con lesiones traumatológicas y ortopédicas que no se puedan postergar; disminuir el uso de recursos para colaborar con el sistema sanitario en general y evitar exposiciones innecesarias. ${ }^{5,14}$

Dividir el Servicio en grupos de trabajo es una medida que disminuye la exposición del personal, las posibilidades de infección cruzada con el resto del equipo y permite realizar una autocuarentena. Farrell y cols. recomiendan disponer de un equipo de respaldo para cuando los grupos estén sobrepasados o se hayan contagiado. ${ }^{2}$

El Hospital de Niños de la ciudad de Córdoba forma parte del polo sanitario que fue destinado a la atención de pacientes con sospecha o diagnóstico de COVID-19. Se restringió la circulación de la población general y se acondicionaron hospitales periféricos para atender a pacientes sin COVID-19.

El Servicio de Ortopedia y Traumatología pediátrica es el único del sistema sanitario público provincial; por lo tanto, habitualmente, recibe derivaciones de todo el territorio. Por este motivo, debió adaptarse a la situación para seguir brindando atención.

Como es de esperar, durante la pandemia, disminuyen las consultas habituales y permanece la demanda ante casos más críticos y serios, como tumores, trauma, infecciones y complicaciones posoperatorias. ${ }^{10}$

Si comparamos la cantidad de consultas externas y cirugías con la del mismo período de 2019, hubo una drástica disminución del $80 \%$. En la bibliografía, también se informa el retraso de la consulta, de manera significativa, hasta en el $35 \%$ de los pacientes. ${ }^{11}$ Este dato no fue contrastado en nuestro estudio.

La institución tomó la decisión de suspender todas las cirugías programadas (aun las ambulatorias), solo estaban permitidas aquellas que, por su retraso, significaran un gran deterioro de la condición del paciente. Con respecto a esto, a diferencia de nuestra institución, algunos centros consideran seguros los procedimientos ambulatorios necesarios, con medidas de protección y no los interrumpieron. ${ }^{1,2,6,15}$

La mayor cantidad de nuestras cirugías en el período estudiado fueron por fracturas supracondíleas. La cantidad se mantuvo constante comparada con la de igual período de 2019.

La cantidad de consultas por Guardia disminuyó un $70 \%$ con respecto a igual período del año anterior. La disminución fue más marcada al comienzo del registro, lo que coincidió con la etapa más dura de confinamiento; se observó un aumento de la demanda y las fracturas acompañando las flexibilizaciones. Esto se demuestra comparando la primera semana de registro (confinamiento estricto con circulación solo del personal esencial), cuando el promedio de atención fue de tres pacientes por día, con la última semana (fase 3 de apertura, salidas recreativas y actividad comercial), que arroja un promedio de 6,43 pacientes diarios.

Si bien es difícil contrastar esto con los reportes mundiales debido a la disparidad de las medidas de restricción aplicadas, varios autores mencionan la disminución de casos durante la pandemia y el confinamiento. ${ }^{2,6,8,11}$

Por el contrario, Hernigou y cols. comunican un aumento del trauma en los niños. También advierten que el confinamiento con familias que se quedan juntas en la casa aumenta las posibilidades de negligencia y fracturas infantiles. ${ }^{12}$

En nuestra serie, solo se registró a un paciente con diagnóstico de fractura humeral y sospecha de maltrato.

Por lejos la gran mayoría de las consultas fueron por traumatismo previo $(86,5 \%)$, seguidas de infecciones y controles posteriores a un tratamiento. Esto también muestra una correlación con las experiencias de otras instituciones. ${ }^{1,5,8,11-13}$

En tiempos de confinamiento, con restricciones a la circulación, no debe llamarnos la atención que el lugar del traumatismo fuera, con diferencia, dentro del domicilio $(56,3 \%) \mathrm{y}$, si incluimos el patio del domicilio, el porcentaje asciende al $72,6 \%$.

Los diagnósticos más habituales no variaron con los de la práctica común, el miembro superior fue el segmento más afectado y las fracturas de muñeca fueron las más frecuentes. ${ }^{16}$

Sí llama la atención la cantidad de fracturas supracondíleas registradas que casi igualaron en número a las fracturas de muñeca ( 23 vs. 24). Es posible que este aumento en la relación con respecto a las estadísticas habituales históricamente publicadas se deba a que otros centros absorbieron parcialmente los casos menores (p. ej., fracturas de muñeca sin desplazamientos).

Muchos centros sugieren el seguimiento por telemedicina. En nuestro medio, no fue posible debido a la falta de infraestructura necesaria para realizarlo. . $2,6,6,10,11,16-19^{-19}$ 


\section{CONCLUSIONES}

En situaciones de emergencia sanitaria, es necesario adaptar, de manera rápida y eficaz, los Servicios, con el fin de colaborar brindando atención, sin aumentar la tensión sobre el sistema sanitario, preservando, de igual manera, al paciente y al personal.

Se recomienda dividir al equipo en grupos de trabajo y fomentar la telemedicina, si es posible.

Si bien la cantidad de pacientes se ve afectada directamente por el confinamiento, se debe estar preparado para seguir atendiendo traumatismos frecuentes en los niños y sus fracturas habituales, como así también estar atento a la aparición de infecciones osteoarticulares y no descuidar la atención de enfermos con cuadros crónicos que no pueda ser postergada.

Conflicto de intereses: Los autores no declaran conflictos de intereses.

ORCID de M. Luna: https://orcid.org/0000-0002-2466-9492

ORCID de L. Zecchini: hittps://orcid.org/0000-0002-2284-3266

\section{BIBLIOGRAFÍA}

1. Service BC, Collins AP, Crespo A, Couto P, Gupta S, Avilucea F, et al. Medically necessary orthopaedic surgery during the COVID-19 pandemic: safe surgical practices and a classification to guide treatment. J Bone Joint Surg Am 2020;102(14):e76. https://doi.org/10.2106/JBJS.20.00599

2. Farrell S, Schaeffer EK, Mulpuri K. Recommendations for the care of pediatric orthopaedic patients during the COVID pandemic. J Am Acad Orthop Surg 2020;28(11):e477-e486. https://doi.org/10.5435/JAAOS-D -20-00391

3. Liang ZC, Chong MSY, Sim MA, Lim JL, Castañeda P, Green DW, et al. Surgical considerations in patients with COVID-19: what orthopaedic surgeons should know. J Bone Joint Surg Am 2020;102(11):e50. https://doi.org/10.2106/JBJS.20.00513

4. Información epidemiológica. Ministerio de Salud de la República Argentina. Disponible en: https://www.argentina.gob.ar/salud/coronavirus-COVID-19/sala-situacion

5. Di Martino A, Faldini C. Trauma service reorganization in Bologna (Italy) during COVID-19 pandemic. Injury 2020;51(7):1684. https://doi.org/10.1016/j.injury.2020.04.033 0020-1383

6. Chang Liang Z, Wang W, Murphy D, Po Hui JH. Novel coronavirus and orthopaedic surgery: early experiences from Singapore. J Bone Joint Surg Am 2020;102(9):745-9. https://doi.org/10.2106/JBJS.20.00236

7. Awad ME, Rumley JCL, Vazquez JA, Devine JG. Perioperative considerations in urgent surgical care of suspected and confirmed coronavirus disease 2019 orthopaedic patients: operating room protocols and recommendations in the current coronavirus disease 2019 pandemic. J Am Acad Orthop Surg 2020;28(11):451-63. https://doi.org/10.5435/JAAOS-D-20-00227

8. Nuñez JH, Sallent A, Lakhani K, Guerra-Farfan E, Vidal N, Ekhtiari S, et al. Impact of the COVID-19 pandemic on an Emergency Traumatology Service: experience at a Tertiary Trauma Centre in Spain. Injury 2020;51(7):1414-8. https://doi.org/10.1016/j.injury.2020.05.016

9. Iyengar K, Vaish A, Vaishya R. Revisiting conservative orthopaedic management of fractures during COVID-19 pandemic. J Clin Orthop Trauma 2020;11(4):718-20. https://doi.org/10.1016/j.jcot.2020.05.010

10. Chhabra HS, Bagaraia V, Keny S, Kalidindi KKV, Mallepally A, Dhillon MS, et al. COVID-19: current knowledge and best practices for orthopaedic surgeons. Ind J Orthop 2020;54(4)1-15. https://doi.org/10.1007/s43465-020-00135-1

11. Luengo-Alonso G, García-Seisdedos Pérez-Tabernero FG, Tovar-Bazaga M, Arguello-Cuenca JM, Calvo E. Critical adjustments in a department of orthopaedics through the COVID-19 pandemic. Int Orthop 2020;44(8):1557-64. https://doi.org/10.1007/s00264-020-04647-1 
12. Hernigou J, Morel X, Callewier A, Bath O1, Hernigou P. Staying home during “COVID-19” decreased fractures, but trauma did not quarantine in one hundred and twelve adults and twenty eight children and the "tsunami of recommendations" could not lockdown twelve elective operations. Int Orthop 2020;44(8):1473-80. https://doi.org/10.1007/s00264-020-04619-5

13. Randelli PS, Compagnoni R. Management of orthopaedic and traumatology patients during the coronavirus disease (COVID-19) pandemic in northern Italy. Knee Surg Sports Traumatol Arthrosc 2020;28(6):1683-9. https://doi.org/10.1007/s00167-020-06023-3

14. Wilson JM, Schwartz AM, Farley KX, Devito DP, Fletcher ND. Doing our part to conserve resources. J Bone Joint Surg Am 2020;102(13):e66. https://doi.org/10.2106/JBJS.20.00567

15. Naranje SN, Erali RA, Warner WC Jr, Sawyer JR, Kelly DM. Epidemiology of pediatric fractures presenting to emergency departments in the United States. J Pediatr Orthop 2016;36(4):e45-8. https://doi.org/10.1097/BPO.0000000000000595

16. Feeley I, McAleese T, Clesham K, Moloney D, Crozier-Shaw G, et al. Foot and ankle service adaptation in response to COVID-19 and beyond. Ann Med Surg (Lond) 2020;54:62-4. https://doi.org/10.1016/j.amsu.2020.04.023

17. Thaler M, Khosravi I, Leithner A, Papagelopoulos PJ, Ruggieri P. Impact of the COVID-19 pandemic on patients suffering from musculoskeletal tumours. Int Orthop 2020;44(8):1503-9. https://doi-org/10.1007/s00264-020-04636-4

18. Ranuccio F, Tarducci L, Familiari F, Mastroianni V, Giuzio E. Disruptive effect of COVID-19 on orthopaedic daily practice. J Bone Joint Surg Am 2020;102(14):e77. https://doi.org/10.2106/JBJS.20.00604

19. Sarac NJ, Sarac BA, Schoenbrunner AR, Janis JE, Harrison RK, Phieffer L, et al. A Review of state guidelines for elective orthopaedic procedures during the COVID-19 outbreak. J Bone Joint Surg Am 2020;102(11):942-5. https://doi.org/10.2106/JBJS.20.00510 